\title{
Advances in Care for Insulin-Requiring Patients Without Closed Loop
}

\author{
Rayhan A. Lal, MD, ${ }^{1,2}$ Bruce Buckingham, MD, and David M. Maahs, MD, PhD ${ }^{1}$
}

Keywords: Non-artificial pancreas review, Insulin formulations, Insulin delivery, Devices, Glucose monitoring, Decision support.

\section{Introduction}

$\mathbf{F}$ OR PEOPLE WITH diabetes, there is growing evidence for the improved safety and glycemic control with artificial pancreas systems. ${ }^{1-2}$ Despite this, the use of hybrid closedloop diabetes technology remains limited. Sherr et al. reported on rates of insulin pump use among children and adolescents with type 1 diabetes enrolled in the German/Austrian Prospective Diabetes Follow-up Registry (DPV), the US T1D Exchange (T1DX), and the English/Welsh National Paediatric Diabetes Audit (NPDA). The NPDA group had only $14 \%$ pump utilization, while DPV and T1DX had greater use of $41 \%$ and $47 \%$, respectively, in 2011-2012. ${ }^{3}$ Another study analyzing an international pediatric diabetes registry of 16,570 found $44 \%$ of participants using insulin pumps. ${ }^{4}$ Tanenbaum analyzed 1503 adult patients from the T1DX and found $32 \%$ used an insulin pump and continuous glucose monitor (CGM), $38 \%$ used an insulin pump with glucometer, 5\% used multiple daily injections (MDI) with CGM, and 25\% used MDI with glucometer. ${ }^{5}$ They also assessed barriers to device use among 249 subjects who discontinued CGM and 72 who stopped using an insulin pump. The most commonly reported obstacles to CGM use were cost, number of alarms, inaccuracy, body image concerns related to wearing devices, and discomfort. The most frequent reasons for stopping insulin pump use were body image with wearing the device, discomfort, cost, and trust. 5

In addition to the ADA-reported 1.25 million people in the United States with type 1 diabetes, about $29 \%$ of the 29 million individuals with type 2 diabetes use insulin. ${ }^{6}$ Indeed, this 8.4 million represents the vast majority of insulin users in the United States. The 2014 OpT2mise international controlled trial of 331 patients with type 2 diabetes on insulin demonstrated significant reduction in hemoglobin A1c (HbA1c) of $0.7 \%(P<0.0001)$ at 6 months for those using insulin pump rather than MDI. ${ }^{7}$ However, the most recent closed-loop technology from Medtronic received FDA approval for only type 1 diabetes. The hybrid closed-loop technology uses continuous glucose data to modulate the delivery of insulin by a pump utilizing a control algorithm. While artificial pancreas technology continues to mature, it is only used by a minority of insulin requiring patients. There remains a significant need to accelerate clinical research and investigate extending indications for insulin requiring individuals. In this section of the supplement, we present other emerging technologies, including novel insulin, insulin delivery devices, glucose monitoring technology, and decision support tools for the population not currently utilizing closedloop insulin delivery systems.

\section{Insulin}

Insulin analogs have been available in the United States since the approval of lispro in 1996. Previously approved rapid-acting insulin analogs (lispro, aspart, and glulisine) and basal insulin analogs (glargine and detemir) have been shown to reduce rates of hypoglycemia and decrease HbA1c in those with diabetes, although some debate the clinical significance of these reductions. ${ }^{8,9}$ Novel insulins continue to be developed, which may improve outcomes or quality of life for those on MDI. In general, a faster acting prandial insulin would better simulate first-phase insulin response and longer acting basal insulins improve steady-state insulin levels.

In June 2014, the FDA approved an insulin inhalation powder (Afrezza) as an ultrarapid-acting insulin in adults with diabetes. ${ }^{10}$ Indeed, Afrezza is faster acting than any subcutaneous insulin formulation. The AFFINITY-1 trial concluded that in patients with type 1 diabetes receiving basal insulin, Afrezza was noninferior to aspart with a reduction in hypoglycemia. ${ }^{11}$ The AFFINITY-2 trial demonstrated that in patients with type 2 diabetes uncontrolled on oral medications, Afrezza taken before meals significantly lowered HbAlc by $0.4 \%(P<0.0001) .^{12}$

\footnotetext{
${ }^{1}$ Division of Endocrinology, Department of Pediatrics, Stanford University School of Medicine, Stanford, California

${ }^{2}$ Division of Endocrinology, Department of Medicine, Stanford University School of Medicine, Stanford, California.
} 
The FDA approved insulin glargine $300 \mathrm{U} / \mathrm{mL}$ (Toujeo) in February 2015. A recent 2017 article by Bergenstal et al. compared $100 \mathrm{U} / \mathrm{mL}$ glargine to Toujeo in 59 adult participants with type 1 diabetes. They found less increase in CGM glucose levels during the last $4 \mathrm{~h}$ of a $24-\mathrm{h}$ injection interval and reduced nocturnal hypoglycemia. ${ }^{13}$

Insulin degludec (Tresiba) is a novel basal insulin with a duration of action of more than $42 \mathrm{~h} .{ }^{14}$ It was FDA approved in September 2015 for use in those with diabetes older than age 1 . This increased duration of action allows for greater flexibility in timing of basal insulin administration, 8-40 h between doses. ${ }^{15,16}$ Systematic review of four studies among 1846 participants with type 1 diabetes demonstrated reduced rates of nocturnal hypoglycemia with similar metabolic control and reduced basal insulin dose. ${ }^{17}$ Additional data from 7637 individuals with type 2 diabetes revealed lower risk of hypoglycemia in the insulin degludec group. ${ }^{18}$ An additional benefit of insulin degludec is that it can be coformulated with insulin aspart (Ryzodeg) and the GLP-1 liraglutide (Xultophy), reducing total daily injections.

Novo Nordisk received FDA approval for a fast-acting insulin aspart (Fiasp) in September 2017. It contains the excipients niacinamide and L-arginine hydrochloride. Recent publications have demonstrated small, $0.1 \%$, but significant $(P=0.0424)$ decreases in HbA1c and 1-h postprandial plasma glucose levels among 381 subjects with type 1 diabetes. ${ }^{19} \mathrm{~A}$ similar modest decrease in 1-h postprandial glucose concentration was observed in a head-to-head trial comparing Fiasp with conventional insulin aspart in 689 participants with type 2 diabetes. There was also an increase in early postprandial hypoglycemia. No HbA1c difference was observed between groups. ${ }^{20}$

\section{Insulin delivery devices}

Subcutaneous delivery of insulin has evolved over time. Syringes are now shorter and thinner than ever before, limited by the structural integrity needed to penetrate a vial and skin. Insulin pens advanced the design and allowed for even smaller needles and more convenient administration. A systematic review conducted by Lasalvia et al. revealed that among adults with type 2 diabetes, insulin pen use was associated with HbA1c closer to goal, fewer episodes of hypoglycemia, and greater adherence to therapy. Of note, all reviewed articles were assessed as having some risk of bias, largely due to the lack of masking and insufficient explanation of randomization methods. ${ }^{21}$

Several new insulin "smartpen" systems integrate the standard insulin pen with additional features. The Eli Lilly Memoir insulin pen was one of the first to track administered doses and can store the last 16 doses administered. It was successfully utilized to obtain insulin administration records for 10 adolescents with type 1 diabetes receiving MDI in addition to CGM, diet, and exercise data. ${ }^{22}$ Another randomized, open-label study of the Memoir pen from the same year evaluated 257 participants with type 1 diabetes and baseline $\mathrm{HbA1c} \geq 8 \%$. The investigators compared glycemic control among one group using the pen with memory function and another group without this function, the Luxura. There was no difference in rates of hypoglycemia or HbAlc. The group concluded that this poorly controlled patient population did not benefit from the memory function but that others might. ${ }^{23}$ Despite these results, many companies have been working with newer technologies to engineer pens that interface with smartphones.

Companion Medical has created the InPen system, which tracks administered doses, provides bolus advice in $0.5 \mathrm{U}$ increments, and can transmit these data via Bluetooth. The Pendiq system sold in Europe and Australia offers $0.1 \mathrm{U}$ dosing, tracks dose and time of administration, and also supports Bluetooth data transmission. Novo Nordisk developed the NovoPen Echo for injection of insulin aspart in $0.5 \mathrm{U}$ increments with internal tracking of administered doses. A 2010 multicenter study by Olsen et al. demonstrated higher satisfaction scores with NovoPen Echo among 205 pediatric patients with type 1 diabetes, their parents, and healthcare providers. ${ }^{24}$ Emperra Esysta system includes an insulin pen that tracks doses and a glucometer, both able to upload data via Bluetooth, passively moving information from patient to healthcare provider. Finally, several systems are in development, which attach to conventional insulin pens or replace the cap. These devices may record the time of the last dose administration, track remaining insulin or dose administered, and relay the information via Bluetooth. ${ }^{25}$ While it seems these devices should improve data logging and accuracy of dosing, randomized controlled trials are needed.

Calibra Medical has developed a 3-day wearable patch system that allows for delivery of mealtime insulin in $2 \mathrm{U}$ increments after squeezing the device. In a clinical trial of 101 adults with type 1 or type 2 diabetes, participants preferred the patch over pens or syringes. ${ }^{26}$

\section{Glucose monitoring}

Despite significant advances in technology, glucose meters remain the primary means of blood sugar assessment among those with diabetes. Modern glucometers require significantly less blood, are easier to use, and more accurate than ever before. Newer glucose meters, such as the Contour Next One, OneTouch Verio Flex, Accu-Chek Aviva Connect, and TrueMetrix Bluetooth blood glucose meter can transmit readings via Bluetooth. Ekhlaspour et al. compared the accuracy of 17 different commercially available glucose meters against Yellow Springs Instrument (YSI) 2300 in 2016. They found a range of accuracies, with mean absolute relative difference (MARD), varying from $5.6 \%$ for the Contour Next to $20.8 \%$ for the Nipro SideKick. ${ }^{27}$ CGM offers accuracy within this range and will soon operate independently of the glucometer.

The continuous glucose monitoring literature often highlights the use of this technology with insulin pumps, but the data suggest effectiveness regardless of method of insulin delivery. Early studies of CGMs in 60 adults with type 1 diabetes showed similar decreases in HbA1c regardless of use with MDI or insulin pump. ${ }^{28}$ The DIAMOND clinical trial randomized 158 adults with type 1 diabetes on MDI to CGM or usual care and found a significant $0.6 \%$ reduction in $\mathrm{HbA} 1 \mathrm{c}$ $(P<0.001)$ following 24 weeks of CGM wear. ${ }^{29}$ Further work from the same group revealed CGM improved A1c and reduced glycemic variability in 116 individuals 60 years and older with type 1 and type 2 diabetes using MDI. ${ }^{30}$ Their most recent publication revealed that among 158 adults with type 2 diabetes on MDI, HbA1c was $0.3 \%$ lower $(P=0.022)$ after 24 weeks of CGM use compared with usual care. ${ }^{31}$

CGM technology continues to advance with the latest Dexcom G6 system, FDA approved in March 2018. This new 
system can be used for diabetes treatment decisions without glucometer (so-called nonadjunctive use), requires no fingerstick calibration, is unaffected by acetaminophen, allows for 10 days of wear, and can transmit data via Bluetooth to a smartphone. ${ }^{32,33}$ With this labeling, CGM technology is poised to replace conventional glucose monitors.

As opposed to continuous glucose monitoring, flash-based glucose monitoring provides buffered glucose data on-demand. While a motivated user can acquire significant data, real-time alarms are only possible at the time of scanning. In September 2017, the FDA approved the Abbott FreeStyle Libre flash glucose monitoring system for use in adults with diabetes to make treatment adjustments without fingerstick calibration. ${ }^{34}$ The IMPACT randomized controlled trial assessed the effect of wearing FreeStyle Libre flash glucose monitoring system in 163 well-controlled individuals with type 1 diabetes. After 6 months they report a reduction in hypoglycemia without significant change in HbA1c. ${ }^{35}$ Haak et al. performed an openlabel randomized controlled study of the FreeStyle Libre on 224 adult participants with type 2 diabetes on insulin for 6 months. At the completion of the study, there was no difference in HbAlc between the groups, but there was a statistically significant reduction in hypoglycemia by $0.47 \mathrm{~h}$ per day $(P=0.0006) .^{36}$

The Senseonics Eversense, 90-day implantable CGM, is still under FDA review at the time of this writing. The PRECISE II trial was a nonrandomized, masked, prospective, single-arm, multicenter study evaluating this new technology against the gold standard YSI reference. The device had an MARD of $8.8 \%$, on par with the most accurate subcutaneous sensors. ${ }^{37}$

\section{Decision support}

Individuals with diabetes must make insulin dosing decisions multiples times per day. These choices require mental effort, including making calculations and attempting to recall past effects to inform present conclusions. In an effort to reduce this continual cognitive demand, industry has attempted to create software that can perform the needed calculations and ultimately use past data to support clinician or patient decision-making.

In December 2002, Smiths Medical introduced the Deltec Cozmo, which was the first insulin pump with an automated bolus calculator. By 2008, most insulin pump manufacturers provided this feature in addition to accounting for insulin-onboard. ${ }^{38}$ Around the same time, Garg et al. performed a 1-year open-label randomized control trial on the use of the AccuChek bolus advisor software running on a Palm personal digital assistant (PDA) for adults with type 1 diabetes on MDI. They showed a statistically significant reduction in $\mathrm{HbA} 1 \mathrm{c}$ for those using the software. ${ }^{39}$

The 2012 BolusCal study of 51 adults with type 1 diabetes and baseline HbA1c 8\%-10.5\% compared placebo to teaching carbohydrate counting with and without a combined glucometer and automated bolus calculator, the Accu-Chek Aviva Expert. Reduction in HbA1c in the carbohydrate counting groups was significantly lower than in the placebo group. Although no difference in HbA1c was reported in those who used the bolus calculator, treatment satisfaction was greater in this group. ${ }^{40}$ The ABACUS trial was a 26 -week prospective, randomized controlled multicenter trial evaluating the Aviva Expert in
193 adults with type 1 and type 2 diabetes. They found significantly more patients utilizing the Accu-Chek system had a $>0.5 \%$ HbAlc reduction without increased hypoglycemia and that the system improved patient satisfaction. ${ }^{41}$ The StenoABC trial confirmed these findings in 130 adults with type 1 diabetes randomized to advanced carbohydrate counting teaching with either mental calculations or Accu-Chek Aviva Expert. While both groups had a statistically significant reduction in $\mathrm{HbA1c}$, the difference was greater in those guided by the bolus calculator. $^{42}$

Since the PDA and glucometer advisor studies, the widespread availability of smartphones has led to a rapid increase in the availability of decision support software (Fig. 1). Early diabetes management software from WellDoc ran on flip phones and incorporated more than 1000 automated educational and motivational messages into a feedback algorithm. A 2011 cluster-randomized trial of the system included 163 participants with type 2 diabetes and a mean $\mathrm{HbA} 1 \mathrm{c}$ of $9.4 \%$. The maximally treated group had an HbA1c reduction of $1.9 \%$, while the control group had a drop of $0.7 \%$. There was no significant difference in diabetes distress, depression, symptomatology, blood pressure, or lipids. ${ }^{43}$

In 2015, Huckvale et al. assessed 46 dose calculators for $\mathrm{iOS}$ and Android. They found that $59 \%$ contained a clinical disclaimer, $30 \%$ documented the formulas used, 91\% lacked numeric input validation, 59\% allowed calculation with missing inputs, $48 \%$ used ambiguous terminology, $9 \%$ lacked numeric precision, and $4 \%$ did not reliably store parameters. Crucially, 67\% carried a risk of inappropriate dose recommendation and only one $\mathrm{iOS}$ app was issue free based on the author's criteria. ${ }^{44}$ Since that time, new apps continue to flood the App Store and Google Play Store. This has led experts in the field to suggest the need for greater scrutiny and standards. ${ }^{45,46}$

There has been a growing body of literature evaluating decision support interventions. Small pilot studies often have inconclusive results and lack follow-up. For example, a 6-week nonrandomized single-arm pilot study of the Advanced Bolus Calculator for Diabetes (ABC4D) in 2016 did not reach statistical significance for postprandial hyperglycemia or hypoglycemia. ${ }^{47}$ A 2016 meta-analysis of 14 studies examining phone apps included 1360 subjects, predominantly adults with type 2 diabetes, and found an average statistically significant $\mathrm{HbA} 1 \mathrm{c}$ reduction of $0.49 \%(P<0.01) .{ }^{48}$ In an effort to review more "modern" diabetes support tools, Greenwood et al. performed a systematic review of 25 studies published between January 2013 and January 2017. Seventy-two percent of these articles reported significant reduction in $\mathrm{HbA1c}$, which was more pronounced in studies involving type 2 diabetes. They report that the key elements for a reduced $\mathrm{HbA1c}$ outcome include the following: health data from the user, two-way communication with the healthcare team, education and feedback. ${ }^{49}$

Multiple new commercial systems continue to enter the market, with more impressive outcome data. The Livongo Diabetes Program offers members a glucometer that connects and uploads data to their servers via a cellular network. A CDE coach is able to contact the user by phone if glucose is outside a threshold range. In one retrospective analysis of 4544 members, this intervention increased time in range during 1 year of use. ${ }^{50}$ The One Drop app utilizes a proprietary glucometer for passive transfer of glucose data or manual entry with other devices. The program provides a 

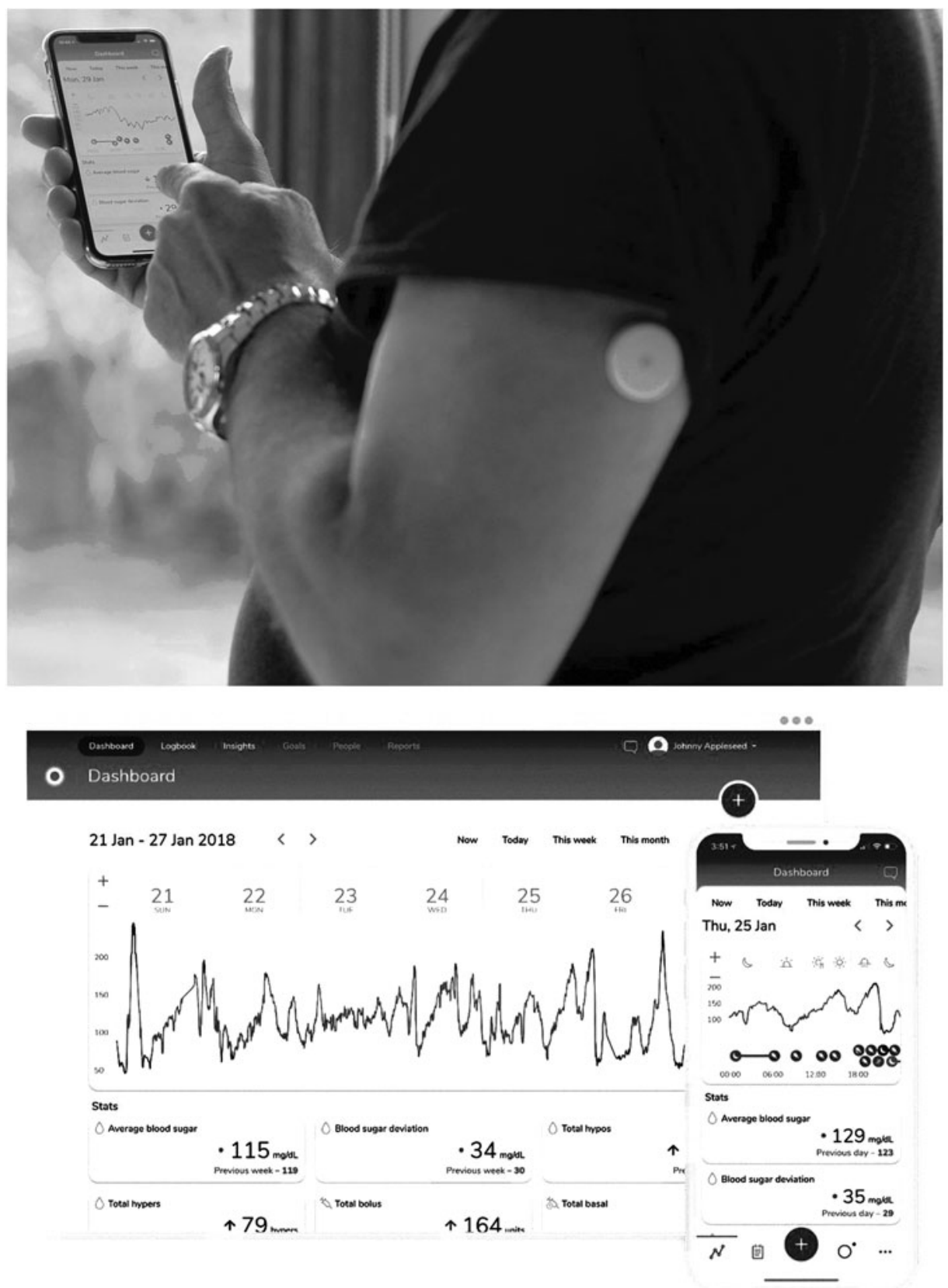

FIG. 1. Diabetes tracking software, with data upload from Abbott FreeStyle Libre. Pictures provided by Center Health.

food library to expedite carbohydrate counting, medication reminders, and motivational messages. In a retrospective review of 256 adult participants with type 1 or type 2 diabetes, using One Drop, there was a $1.36 \%$ decrease in HbA1c $(P<0.001)$ over 1 year, independently associated with using the program to track carbohydrates. ${ }^{51}$ The Glooko Mobile App allows users to upload data from more than 50 glucometers. Offringa et al. compared 899 individuals using Glooko with 900 control participants with type 1 and type 2 diabetes. They found a statistically significant increase of 8.8 tests per month $(P<0.001)$ among app users, as well as fewer hyperglycemic events and lower average glucose. ${ }^{52}$

The regulatory landscape for clinical decision support tools is currently changing. In December 2017, the FDA released draft guidelines for Clinical and Patient Decision
Support Software. It proposed that software visualizing data or aiding healthcare professional decision-making should be under less scrutiny than patient decision support software. Industry and professional organizations supporting widespread and rapid use of patient decision support have criticized the draft guidelines. Presently, many of the newly developed tools avoid advising patients to escape regulatory hurdles.

\section{Future directions}

Significant resources are being spent on the development of closed-loop insulin delivery systems. However, only a minority of individuals with diabetes are currently using these systems. Commonly reported reasons for discontinuing insulin pump and GGM therapy include perceived body 
image, cost, and discomfort. ${ }^{5}$ Other avenues of innovation are required for the millions who do not possess artificial pancreas technology.

The widespread availability of smartphones with Bluetooth, global positioning system (GPS), and fitness trackers makes them ideal vehicles for passively collecting data from compatible diabetes technology. Currently, these data can be used by the patient and healthcare provider to help guide changes in treatment. Because there is less regulation of data transmission without decision support, this information can be incorporated into a remote telehealth visit with an endocrinologist. $^{53}$

Passively moving data to physicians for analysis is an area of active research. Ideally, data can be sent via Bluetooth to a smartphone app, which can then be exported to the electronic medical record system without patient intervention. ${ }^{54}$ A significant benefit of passive data collection from connected devices is that each device does not require special equipment to download. This significantly reduces time in clinic spent obtaining the information from glucometer, CGM, insulin pumps, or smart pens. A future challenge will be creating an agreed on format for all this information. Uniformity will allow for streamlined data mining and can provide valuable observational data at the population level.

DreaMed Diabetes recently received regulatory approval in Europe for its Advisor Pro product, which assists clinicians in optimizing insulin therapy for their patients. The system uses proprietary algorithms, including machine learning and fuzzy logic, to process data from downloads of CGM, glucometer, and insulin pump. The healthcare provider will receive a patient report with recommendations and can then edit and share the suggestions. ${ }^{55}$ While the system is still in clinical trial, it provides a model for future digital clinics delivering personalized precision care.

Once regulatory hurdles are overcome, then patient decision support becomes viable. With sufficient data collection, computational techniques may be applied to guide management in a personalized manner. Significant work is needed to ensure the safety of these platforms. The benefits include empiric, personalized recommendations that incorporate data from the multitude of sensors that we now routinely carry in our pocket.

\section{Acknowledgments}

Dr. Lal is a Stephen Bechtel Endowed Adult and Pediatric Endocrinology Fellow through the Stanford Child Health Research Institute and is supported by a Diabetes, Endocrinology, and Metabolism Training Grant (T32 DK007217) from NIDDK.

\section{Author Disclosure Statement}

Dr. Buckingham has research support from the NIH (DP3DK104059, DP3DK101055, DK-14-024), Helmsley Foundation, Medtronic Diabetes, Insulet, Dexcom, Xeris, Roche, Bigfoot Biomedical, and Tandem. He is on advisory boards for Novo Nordisk, ConvaTec, BD, and Insulet and has consulted for Tandem.

Dr. Maahs has research support from the NIH (including P30DK116074), JDRF, NSF, and the Helmsley Charitable Trust and his institution has research support from Medtronic, Dexcom, Insulet, Bigfoot Biomedical, and Roche.
Dr. Maahs has consulted for Abbott, the Helmsley Charitable Trust, Sanofi, and Eli Lilly and has served on an advisory board for Insulet.

\section{References}

1. Garg SK, Weinzimer SA, Tamborlane WV, et al.: Glucose outcomes with the in-home use of a hybrid closed-loop insulin delivery system in adolescents and adults with type 1 diabetes. Diabetes Technol Ther 2017;19:155-163.

2. Bergenstal RM, Garg S, Weinzimer SA, et al.: Safety of a hybrid closed-loop insulin delivery system in patients with type 1 diabetes. JAMA 2016;316:1407-1408.

3. Sherr JL, Hermann JM, Campbell F, et al.: Use of insulin pump therapy in children and adolescents with type $1 \mathrm{di}-$ abetes and its impact on metabolic control: comparison of results from three large, transatlantic paediatric registries. Diabetologia 2016;59:87-91.

4. Szypowska A, Schwandt A, Svensson J, et al.: Insulin pump therapy in children with type 1 diabetes: analysis of data from the SWEET registry. Pediatr Diabetes 2016;17 Suppl 23:38-45.

5. Tanenbaum ML, Hanes SJ, Miller KM, et al.: Diabetes device use in adults with type 1 diabetes: barriers to uptake and potential intervention targets. Diabetes Care 2017;40: 181-187.

6. Selvin E, Parrinello CM, Daya N, Bergenstal RM: Trends in insulin use and diabetes control in the U.S.: 1988-1994 and 1999-2012. Diabetes Care 2016;39:e33-e35.

7. Reznik Y, Cohen O, Aronson R, et al.: Insulin pump treatment compared with multiple daily injections for treatment of type 2 diabetes (OpT2mise): a randomised open-label controlled trial. Lancet 2014;384:1265-1272.

8. Grunberger G: Insulin analogs-are they worth it? Yes! Diabetes Care 2014;37:1767-1770.

9. Davidson MB: Insulin analogs-is there a compelling case to use them? No! Diabetes Care 2014;37:1771-1774.

10. Fala L: Afrezza (insulin human) inhalation powder approved for the treatment of patients with type 1 or type 2 diabetes. Am Health Drug Benefits 2015;8(Spec Feature): 40-43.

11. Bode BW, McGill JB, Lorber DL, et al.: Inhaled technosphere insulin compared with injected prandial insulin in type 1 diabetes: a randomized 24-week trial. Diabetes Care 2015;38:2266-2273.

12. Rosenstock J, Franco D, Korpachev V, et al.: Inhaled technosphere insulin versus inhaled technosphere placebo in insulin-naïve subjects with type 2 diabetes inadequately controlled on oral antidiabetes agents. Diabetes Care 2015; 38:2274-2281.

13. Bergenstal RM, Bailey TS, Rodbard D, et al.: Comparison of insulin glargine 300 units $/ \mathrm{mL}$ and 100 units $/ \mathrm{mL}$ in adults with type 1 diabetes: continuous glucose monitoring profiles and variability using morning or evening injections. Diabetes Care 2017;40:554-560.

14. Kurtzhals P, Heise T, Strauss HM, et al.: Multi-hexamer formation is the underlying mechanism behind the ultra-long glucose-lowering effect of insulin degludec. Paper presented at: American Diabetes Association (ADA) 71st Scientific Session; Jun 2011; San Diego, CA.

15. Mathieu C, Hollander P, Miranda-Palma B, et al.: NN1250-NN3770 (BEGIN: Flex T1) trial investigators. Efficacy and safety of insulin degludec in a flexible dosing regimen vs insulin glargine in patients with type 1 diabetes (BEGIN: Flex T1): a 26-week randomized, treat-to-target 
trial with a 26-week extension. J Clin Endocrinol Metab 2013;98:1154-1162.

16. Meneghini L, Atkin SL, Gough SC, et al.: NN1250-NN3668 (BEGIN FLEX) trial investigators. The efficacy and safety of insulin degludec given in variable once-daily dosing intervals compared with insulin glargine and insulin degludec dosed at the same time daily: a 26-week, randomized, openlabel, parallel-group, treat-to-target trial in individuals with type 2 diabetes. Diabetes Care 2013;36:858-864.

17. Dżygało K, Golicki D, Kowalska A, et al.: The beneficial effect of insulin degludec on nocturnal hypoglycaemia and insulin dose in type 1 diabetic patients: a systematic review and meta-analysis of randomised trials. Acta Diabetol 2015;52:231-238.

18. Marso SP, McGuire DK, Zinman B, et al.: Efficacy and safety of degludec versus glargine in type 2 diabetes. $\mathrm{N}$ Engl J Med 2017;377:723-732.

19. Mathieu C, Bode BW, Franek E, et al.: Efficacy and safety of fast-acting insulin aspart in comparison with insulin aspart in type 1 diabetes (onset 1): a 52-week, randomized, treat-to-target, phase 3 trial. Diabetes Obes Metab 2018;20: 1148-1155.

20. Bowering K, Case C, Harvey J, et al.: Faster aspart versus insulin aspart as part of a basal-bolus regimen in inadequately controlled type 2 diabetes: the onset 2 trial. Diabetes Care 2017;40:951-957.

21. Lasalvia P, Barahona-Correa JE, Romero-Alvernia DM, et al.: Pen devices for insulin self-administration compared with needle and vial: systematic review of the literature and meta-analysis. J Diabetes Sci Technol 2016;10:959-966.

22. Maahs DM, Mayer-Davis E, Bishop FK, et al.: Outpatient assessment of determinants of glucose excursions in adolescents with type 1 diabetes: proof of concept. Diabetes Technol Ther 2012;14:658-664.

23. Danne T, Forst T, Deinhard J, et al.: No effect of insulin pen with memory function on glycemic control in a patient cohort with poorly controlled type 1 diabetes: a randomized open-label study. J Diabetes Sci Technol 2012;6:1392-1397.

24. Reynholds C, Antal Z: Analysis of the NovoPen ${ }^{\circledR}$ Echo for the delivery of insulin: a comparison of usability, functionality, and preference among pediatric subjects, their parents, and health care professionals. J Diabetes Sci Technol 2010;4: 1476-1478.

25. Bailey TS, Stone JY: A novel pen-based Bluetooth-enabled insulin delivery system with insulin dose tracking and advice. Expert Opin Drug Deliv 2017;14:697-703.

26. Peyrot M, Dreon D, Zraick V, et al.: Patient perceptions and preferences for a mealtime insulin delivery patch. Diabetes Ther 2018;9:297-307.

27. Ekhlaspour L, Mondesir D, Lautsch N, et al.: Comparative accuracy of 17 point-of-care glucose meters. J Diabetes Sci Technol 2017;11:558-566.

28. Garg SK, Voelmle MK, Beatson CR, et al.: Use of continuous glucose monitoring in subjects with type 1 diabetes on multiple daily injections versus continuous subcutaneous insulin infusion therapy: a prospective 6-month study. Diabetes Care 2011;34:574-579.

29. Beck RW, Riddlesworth T, Ruedy K, et al.: Effect of continuous glucose monitoring on glycemic control in adults with type 1 diabetes using insulin injections: the DIAMOND randomized clinical trial. JAMA 2017;317:371-378.

30. Ruedy KJ, Parkin CG, Riddlesworth TD: Continuous glucose monitoring in older adults with type 1 and type 2 diabetes using multiple daily injections of insulin: results from the DIAMOND trial. J Diabetes Sci Technol 2017; 11:1138-1146.

31. Beck RW, Riddlesworth TD, Ruedy K, et al.: Continuous glucose monitoring versus usual care in patients with type 2 diabetes receiving multiple daily insulin injections: a randomized trial. Ann Intern Med 2017;167:365-374.

32. Calhoun P, Johnson TK, Hughes J, et al.: Resistance to acetaminophen interference in a novel continuous glucose monitoring system. J Diabetes Sci Technol 2018;12:393396.

33. Brown A, Close K: FDA Clears Dexcom G6 CGM with No Fingersticks! Launch Later This Year. DiaTribe N.p., March 29, 2018. https://diatribe.org/fda-clears-dexcom-g6-cgm-nofingersticks-launch-later-year (accessed April 10, 2018).

34. Aleppo G, Ruedy KJ, Riddlesworth TD, et al.: REPLACEBG: a randomized trial comparing continuous glucose monitoring with and without routine blood glucose monitoring in adults with well-controlled type 1 diabetes. Diabetes Care 2017;40:538-545.

35. Oskarsson P, Antuna R, Geelhoed-Duijvestijn P, et al.: Impact of flash glucose monitoring on hypoglycaemia in adults with type 1 diabetes managed with multiple daily injection therapy: a pre-specified subgroup analysis of the IMPACT randomised controlled trial. Diabetologia 2018; 61:539-550.

36. Haak T, Hanaire H, Ajjan R, et al.: Flash glucose-sensing technology as a replacement for blood glucose monitoring for the management of insulin-treated type 2 diabetes: a multicenter, open-label randomized controlled trial. Diabetes Ther 2017;8:55-73.

37. Christiansen MP, Klaff LJ, Brazg R, et al.: A prospective multicenter evaluation of the accuracy of a novel implanted continuous glucose sensor: PRECISE II. Diabetes Technol Ther 2018;20:197-206.

38. Zisser H, Robinson L, Bevier W, et al.: Bolus calculator: a review of four "smart" insulin pumps. Diabetes Technol Ther 2008;10:441-444.

39. Garg SK, Bookout TR, McFann KK, et al.: Improved glycemic control in intensively treated adult subjects with type 1 diabetes using insulin guidance software. Diabetes Technol Ther 2008;10:369-375.

40. Schmidt S, Meldgaard M, Serifovski N, et al.: Use of an automated bolus calculator in MDI-treated type 1 diabetes: the BolusCal Study, a randomized controlled pilot study. Diabetes Care 2012;35:984-990.

41. Ziegler R, Cavan DA, Cranston I, et al.: Use of an insulin bolus advisor improves glycemic control in multiple daily insulin injection (MDI) therapy patients with suboptimal glycemic control: first results from the ABACUS trial. Diabetes Care 2013;36:3613-3619.

42. Hommel E, Schmidt S, Vistisen D, et al.: Effects of advanced carbohydrate counting guided by an automated bolus calculator in type 1 diabetes mellitus (StenoABC): a 12-month, randomized clinical trial. Diabet Med 2017;34: 708-715.

43. Quinn CC, Shardell MD, Terrin ML, et al.: Clusterrandomized trial of a mobile phone personalized behavioral intervention for blood glucose control. Diabetes Care 2011; 34:1934-1942.

44. Huckvale K, Adomaviciute S, Prieto JT, et al.: Smartphone apps for calculating insulin dose: a systematic assessment. BMC Med 2015;13:106.

45. Hirsch IB, Parkin CG: Unknown safety and efficacy of smartphone bolus calculator apps puts patients at risk for 
severe adverse outcomes. J Diabetes Sci Technol 2016;10: 977-980.

46. Walsh J, Freckmann G, Roberts R, Heinemann L: Bolus calculator safety mandates a need for standards. J Diabetes Sci Technol 2017;11:3-6.

47. Reddy M, Pesl P, Xenou M, et al.: Clinical safety and feasibility of the advanced bolus calculator for type 1 diabetes based on case-based reasoning: a 6-week nonrandomized single-arm pilot study. Diabetes Technol Ther 2016;18: 487-493.

48. Hou C, Carter B, Hewitt J, et al.: Do mobile phone applications improve glycemic control (HbA1c) in the selfmanagement of diabetes? A systematic review, meta-analysis, and GRADE of 14 randomized trials. Diabetes Care 2016;39: 2089-2095.

49. Greenwood DA, Gee PM, Fatkin KJ, Peeples M: A systematic review of reviews evaluating technology-enabled diabetes self-management education and support. J Diabetes Sci Technol 2017;11:1015-1027.

50. Downing J, Bollyky J, Schneider J: Use of a connected glucose meter and certified diabetes educator coaching to decrease the likelihood of abnormal blood glucose excursions: the Livongo for Diabetes Program. J Med Internet Res 2017;19:e234.

51. Osborn CY, van Ginkel JR, Marrero DG, et al.: One Drop | Mobile on iPhone and Apple Watch: an Evaluation of HbA1c Improvement Associated With Tracking Self-Care. JMIR Mhealth Uhealth 2017;5:e179.
52. Offringa R, Sheng T, Parks L, et al.: Digital diabetes management application improves glycemic outcomes in people with type 1 and type 2 diabetes. J Diabetes Sci Technol 2018; 12:701-708.

53. Prahalad P, Tanenbaum M, Hood K, Maahs DM: Diabetes technology: improving care, improving patient-reported outcomes and preventing complications in young people with type 1 diabetes. Diabet Med 2018;35:419-429.

54. Kumar RB, Goren ND, Stark DE, et al.: Automated integration of continuous glucose monitor data in the electronic health record using consumer technology. J Am Med Inform Assoc. 2016;23:532-537.

55. Sarah F. DreaMed Diabetes Wins CE Mark for Insulin Therapy Management Platform. Drug Delivery Business. February 15, 2018. https://www.drugdeliverybusiness.com/ dreamed-diabetes-wins-ce-mark-insulin-therapy-managementplatform/ (accessed April 10, 2018).

Address correspondence to:

Rayhan A. Lal, MD

Stanford University School of Medicine

Room G-313 Medical Center 300 Pasteur Drive

Stanford, CA 94305-5208

E-mail: inforay@stanford.edu 